\title{
Erratum
}

\section{Principal bundles admitting a rational section}

\section{M.S. Raghunathan}

Invent. math. 116, 409-423(1994)

In the paper referred to in the title, the argument given in $4.10(\mathrm{p} \mathrm{421)}$ in erroneous. It is asserted that the inclusion of $k[Z, \underline{X}]$ in $R$ maps $k$ diagonally in $R$. What one is interested in is the inclusion of $K[Z]$ in $R$ (the natural map of $k[Z, \underline{X}]$ in $R$ is not an injection). But there is a more serious error here: the inclusion of $K[Z]$ in $R$ does not (in the case we are interested in) map $k$ diagonally into $A$ as claimed. Consequently one cannot apply Corollary 1.6 as is done immediately after this. However a modified (and stronger) version of Corollary 1.6 is true and may be applied to rectify the proof. We want to assert that Corollary 1.6 holds even if one equips each $R_{i}[[\underline{Z}]]$ with a $k$-algebra structure such that the composite inclusion $k \hookrightarrow R_{i}[[\underline{Z}]] \rightarrow R_{l}, R_{t}[[\underline{Z}]] \rightarrow R_{l}$ being the standard surjection, is the inclusion of $k$ in $R_{i}$ that gives the given $k$-algebra structure on $R_{i}$. In fact in the entire section $\S 1$, all the results hold when we equip $R[[Z]]$ with any $k$-algebra structure such that the composite map $k \hookrightarrow R[[Z]] \rightarrow R, R[[Z]] \rightarrow R$ being the standard projection, is a $k$-algebra morphism. We have not anywhere in our proofs utilised the assumption that the $k$-algebra structure on $R[[Z]]$ is obtained from the $k$-algebra structure on $R$ through the standard inclusion of $R$ in $R[[Z]]$. Correspondingly, the corollary also holds if the $R_{i}[[\underline{\dot{Z}}]], 1 \leqq i \leqq t$, are equipped with $k$-algebra structures such that the composite maps $k \hookrightarrow R_{i}[[\underline{Z}]] \rightarrow R_{i}$ is the inclusion of $k$ in $R_{l}$ giving the $k$-algebra structure on $R_{i}$. One needs only the fact that the valuation takes the value 0 for all $x \in k, x \neq 0$, which holds even under the weaker condition above.

I thank M. Ojanguren and J.L. Colliot-Thèléne for bringing the error to my attention. 